\title{
Clinical Application of Modified Burns Wean Assessment Program Scores at First Spontaneous Breathing Trial in Weaning Patients from Mechanical Ventilation
}

\author{
Eun Suk Jeong', Kwangha Lee ${ }^{2}$ \\ ${ }^{1}$ Department of Nursing, Pusan National University Hospital, Busan; ${ }^{2}$ Division of Pulmonary, Allergy and Critical Care Medicine, Department of Internal \\ Medicine, Pusan National University School of Medicine, Busan, Korea
}

Background: The purpose of this study was to evaluate the clinical application of modified Burns Wean Assessment Program (m-BWAP) scoring at first spontaneous breathing trial (SBT) as a predictor of successful liberation from mechanical ventilation (MV) in patients with endotracheal intubation.

Methods: Patients requiring MV for more than 72 hours and undergoing more than one SBT in a medical intensive care unit (ICU) were prospectively enrolled over a 3-year period. The m-BWAP score at first SBT was obtained by a critical care nursing practitioner.

Results: A total of 103 subjects were included in this study. Their median age was 69 years (range, 22 to 87 years) and 72 subjects (69.9\%) were male. The median duration from admission to first SBT was 5 days (range, 3 to 26 days), and the rate of final successful liberation from MV was $84.5 \%(n=87)$. In the total group of patients, the successful liberation from MV group at first SBT $(n=65)$ had significantly higher $m$-BWAP scores than did the unsuccessful group (median, 60; range, 43 to 80 vs. median, 53; range, 33 to 70; $P<0.001$ ). Also, the area under the m-BWAP curve for predicting successful liberation of MV was 0.748 (95\% confidence interval, 0.650 to 0.847 ), while the cutoff value based on Youden's index was 53 (sensitivity, 76\%; specificity, 64\%).

Conclusions: The present data show that the m-BWAP score represents a good predictor of weaning success in patients with an endotracheal tube in place at first SBT.

Key Words: endotracheal intubation; intensive care unit; mechanical ventilator; ventilator weaning

\section{INTRODUCTION}

Mechanical ventilation (MV) is a life-saving procedure performed in patients with acute respiratory failure, but the principal goal of critical care management is ultimately to wean patients off respiratory support as soon as possible. Weaning failure is strongly associated with prolonged intensive care unit (ICU) stay and an increased risk of hospital mortality [1,2]. Therefore, predicting the likely success of liberation from MV is an important issue for nurses and physicians. Although many weaning parameters and predictors have been reported in the

\section{Original Article}

Received: August 20, 2018

Revised: October 26, 2018

Accepted: November 5, 2018

Corresponding author

Kwangha Lee

Division of Pulmonary, Allergy, and

Critical Care Medicine, Department

of Internal Medicine, Pusan National

University School of Medicine, 179

Gudeok-ro, Seo-gu, Busan 49241,

Korea

Tel: +82-51-240-7743

Fax: +82-52-245-3127

E-mail: jubilate@pusan.ac.kr

Copyright $\odot 2018$ The Korean Society of Critical Care Medicine

This is an Open Access article distributed under the terms of Creative Attributions Non-Commercial License (http:// creativecommons.org/li-censes/by-nc/4.0/) which permits unrestricted noncommercial use, distribution, and reproduction in any medium, provided the original work is properly cited. 
literature, there is no consensus on the most useful predictive indicators [3-7].

As a comprehensive clinical weaning checklist and scoring instrument, the Burns Wean Assessment Program (BWAP) score was designed to assist clinicians in the management of patients who require long-term MV (LTMV) [8]. Some studies have demonstrated the effectiveness of the BWAP score as a systematic weaning assessment tool in patients requiring LTMV as well as in those receiving MV for more than 3 days [9-11], suggesting that the BWAP score may be helpful when determining ventilator weaning potential. A modified version of the BWAP score (m-BWAP) has been developed, which makes the scoring more objective and convenient [12]. Also, a previous study proposed that m-BWAP is a good indicator of weaning and extubation outcome in patients with LTMV [12].

Many studies have demonstrated successful critical care nurse-directed weaning from MV using well-defined protocols [13-16]. Also, advanced practice nurses can play an important role by weaning patients from MV using well-defined protocols, thereby expediting the weaning process. However, it is still questionable as to whether the m-BWAP instrument could be applied successfully in many Asian ICUs, because the staffing of nurses and full-time intensivists in Korean ICUs of university and teaching hospitals is less robust in comparison with in Western units $[17,18]$, which may delay the recognition of patients capable of being weaned and prolong the duration of MV. Therefore, we hypothesized that the m-BWAP scoring instrument could be applicable for critical care nurses who could drive the initiation of spontaneous breathing trials (SBTs) in appropriate patients.

In this study, we investigated whether the m-BWAP scoring instrument utilized by a critical care nurse practitioner in Korea at first SBT could be applicable to predict successful liberation from MV in patients with endotracheal intubation who have received MV for more than 72 hours.

\section{MATERIALS AND METHODS}

\section{Study Design and Subject Selection}

This prospective, observational study was conducted in a 12bed medical ICU at a 1,300-bed university-affiliated tertiary care hospital in Korea. The ICU has full cardiovascular facilities and close airway monitoring. The physician staff in the medical ICU included one full-time ICU specialist, one clinical fellow (pulmonary and critical care medicine), and one resident physician (internal medicine); the nurse to bed ratio is 1:3. All subjects are managed according to therapeutic rec-

\section{KEY MESSAGES}

- As a comprehensive clinical weaning scoring instrument, the modified Burns Wean Assessment Program (m-BWAP) score was designed as a systematic weaning assessment tool and subsequently proposed as an indicator of weaning outcome in patients having long-term ventilator care. - When predicting ventilator weaning success at first spontaneous breathing trial (SBT), the m-BWAP score (administered by a critical care nurse practitioner) is useful in patients with an endotracheal tube at first SBT.

- Our findings suggest that the m-BWAP score is a useful tool that supports decision-making regarding weaning from mechanical ventilation in Korea.

ommendations, which are based on a lung-protective ventilator strategy [19]. A ventilator weaning protocol, based on documented guidelines, has been developed [20-22], and successfully implemented in our medical ICU (Figure 1).

Between October 1, 2014, and September 30, 2017, adult subjects admitted to the medical ICU and receiving MV for more than 72 hours were screened daily by a critical care nurse practitioner of our medical ICU, who was an independently trained advanced practice nurse. All subjects with more than one SBT were enrolled into the study based on our weaning protocol (Figure 1). Subjects aged younger than 18 years and those with irreversible brain injury, acute or chronic neuromuscular diseases, LTMV prior to ICU admission, and insufficient data on characteristics or outcomes were excluded. Also, patients who received tracheostomy before the first SBT were excluded.

The study protocol was approved by the Institutional Review Board of Pusan National University Hospital (IRB No. H-1409-009-021). Because of the observational nature of this study, the need for informed consent from enrolled subjects or their surrogates was waived. This study had no impact on the treatment of the enrolled patients.

\section{Data Collection and Definition}

The following demographic and clinical data were gathered from the medical records of each subject: age, sex, body mass index (BMI), ICU admission diagnosis, and comorbidities prior to ICU admission (based on the Charlson comorbidity index) [23]. The severity of illness within 24 hours of ICU admission was measured using the Acute Physiology and Chronic Health Evaluation II score, and concomitant organ failure was measured according to the Sequential Organ Failure Assess- 


\begin{tabular}{|c|c|c|}
\hline \multicolumn{3}{|l|}{ Weaning protocol } \\
\hline & Yes & No \\
\hline \multicolumn{3}{|l|}{ A. Daily screening for readiness to wean } \\
\hline \multirow[t]{2}{*}{ 1) Treat underlying cause of respiratory failure } & $\square$ & $\square$ \\
\hline & go to 'B' & reassessed next day \\
\hline \multicolumn{3}{|l|}{ B. Spontaneous awakening trial safety screen } \\
\hline 1) Active seizures & $\square$ & $\square$ \\
\hline 2) Alcohol withdrawal & $\square$ & $\square$ \\
\hline 3) Agitation & $\square$ & $\square$ \\
\hline 4) Paralytics & $\square$ & $\square$ \\
\hline 5) Myocardial ischemia & $\square$ & $\square$ \\
\hline 6) Normal intracranial pressure & $\square$ & $\square$ \\
\hline \multicolumn{2}{|c|}{ one or more Yes (reassessed next day) } & all No (go to 'C') \\
\hline \multicolumn{3}{|l|}{ C. Perform spontaneous awakening trial } \\
\hline 1) Anxiety & $\square$ & $\square$ \\
\hline 2) Agitation & $\square$ & $\square$ \\
\hline 3) Pain & $\square$ & $\square$ \\
\hline 4) Respiration rate $>35$ breaths $/ \mathrm{min}$ for $\geq 5 \mathrm{~min}$ & $\square$ & $\square$ \\
\hline 5) $\mathrm{SpO}_{2}<88 \%$ for $\geq 5 \mathrm{~min}$ & $\square$ & $\square$ \\
\hline 6) Acute cardiac arrhythmia & $\square$ & $\square$ \\
\hline 7) Two or more signs of respiratory distress & $\square$ & $\square$ \\
\hline \multicolumn{3}{|c|}{ ( $60>H R>120$, use of accessory muscles, abdominal paradox, diaphoresis, marked dyspnea) } \\
\hline \multicolumn{2}{|c|}{ one or more Yes (reassessed next day) } & all No (go to 'D') \\
\hline \multicolumn{3}{|l|}{ D. Spontaneous breathing trial safety screen } \\
\hline \multicolumn{3}{|l|}{ 1) Adequate oxygenation } \\
\hline (1) $\mathrm{PaO}_{2} / \mathrm{FiO}_{2}<150 \mathrm{mmHg}$ or $\mathrm{SpO}_{2}<88 \%$ on $\mathrm{FiO}_{2} \leq 0.4$ and $\mathrm{PEEP} \leq 5, \mathrm{Pi}<10$ & $\square$ & $\square$ \\
\hline (2) $\mathrm{pH}<7.25$ or $\mathrm{PaCO}_{2}$ abnormal & $\square$ & $\square$ \\
\hline (3) Respiration rate $>35$ breaths/min & $\square$ & $\square$ \\
\hline (4) Rapid shallow breathing index > 105: & $\square$ & $\square$ \\
\hline \multicolumn{3}{|l|}{ measured on $5 \mathrm{cmH}_{2} \mathrm{O} \mathrm{CPAP}$ (continuous positive airway pressure) for 3 minutes } \\
\hline \multicolumn{3}{|l|}{ 2) Hemodynamic stability } \\
\hline (1) Active myocardial ischemia (chest pain, ST changes, new onset arrhythmia) & $\square$ & $\square$ \\
\hline $\begin{array}{l}\text { (2) Clinically important hypotension (>5 mcg/ } \mathrm{kg} / \mathrm{min} \text { dopamine or dobutamine, } \\
>0.04 \mathrm{mcg} / \mathrm{kg} / \mathrm{min} \text { norepinephrine) }\end{array}$ & $\square$ & $\square$ \\
\hline (3) Heart rate $>140 \mathrm{bpm}$ & $\square$ & $\square$ \\
\hline (4) Fever $\left(>38^{\circ} \mathrm{C}\right)$ & $\square$ & $\square$ \\
\hline \multicolumn{2}{|c|}{ One or more Yes (reassessed next day) } & all No (go to 'E') \\
\hline \multicolumn{3}{|l|}{ E. Perform spontaneous breathing trial (during 30-120 min) } \\
\hline 1) Respiratory rate $>35$ breaths/min, change in $>50 \%$ & $\square$ & $\square$ \\
\hline 2) Heart rate $>120$ bpm, change in $>20 \%$ & $\square$ & $\square$ \\
\hline 3) $90 \mathrm{mmHg}>$ systolic blood pressure $>180 \mathrm{mmHg}$, change in $>20 \%$ & $\square$ & $\square$ \\
\hline 4) Two or more signs of respiratory distress & $\square$ & $\square$ \\
\hline \multicolumn{3}{|l|}{ (Use of accessory muscles, abdominal paradox, diaphoresis, marked dyspnea) } \\
\hline one or & ext day) & all No (go to 'F') \\
\hline F. Physician to consider extubation & & \\
\hline
\end{tabular}

Figure 1. Ventilator weaning protocol. $\mathrm{SpO}_{2}$ : blood oxygen saturation; $\mathrm{HR}$ : heart rate; $\mathrm{PaO}_{2}$ : partial pressure of oxygen; FiO ${ }_{2}$ : fraction of inspired oxygen; $\mathrm{PaCO}_{2}$ : partial pressure of carbon dioxide; PEEP: positive end-expiratory pressure; Pi: inspiratory pressure; ST: ST segment on electrocardiogram. 
ment score $[24,25]$. ICU and hospital length of stay, duration of MV, ICU and hospital cumulative mortality, and tracheostomy status during the ICU stay were also recorded.

The m-BWAP checklists, as reported previously [12], were used in this study, as the m-BWAP score was considered to be easier to measure in subjects prior to SBT than the original version [8]. Enrolled subjects were grouped according to the success of the first SBT, i.e., into successful and unsuccessful liberation from MV groups, respectively. We also evaluated traditional weaning parameters (i.e., rapid shallow breathing index [RSBI], maximal inspiratory pressure [Pimax]) at first SBT to compare predicting successful liberation at first SBT with m-BWAP score. The primary outcome was successful liberation from MV at first SBT, and successful liberation was defined with complete weaning from MV lasting more than 48 hours, regardless of extubation.

\section{Statistical Analysis}

Continuous variables are expressed as median (range), while categorical variables are presented as number (\%). The Student t-test or the Mann-Whitney U-test were used for comparisons of continuous variables according to the normality of the respective distribution. The chi-square or Fisher exact test (for small numbers) was used to compare categorical variables. To estimate the performance of the m-BWAP score in terms of predicting successful weaning from MV, receiver operating characteristic curves were constructed and the area under the curve (AUC) was determined; identification of an optimal cutoff value for this score was based on the maximum Youden's index [26]. All tests of significance were two-tailed, and P-values of $<0.05$ were considered to be statistically significant. All analyses were performed using the IBM SPSS version 24.0 (IBM Corp., Armonk, NY, USA) and MedCalc version 18.5 (MedCalc Software, Ostend, Belgium).

\section{RESULTS}

During the study period, 1,114 subjects were admitted to the medical ICU. Of these, 421 patients $(37.8 \%)$ received MV for more than 72 hours. After the criteria defined above were applied, 103 patients were included in the analyses (Figure 2). The median age of the cohort was 69 years (range, 22 to 87 years) and 72 (69.9\%) were male. Table 1 shows the demographic and clinical characteristics of the study group and a comparison of patients with final successful and unsuccessful liberation from MV, regardless of the number of SBTs. Patients

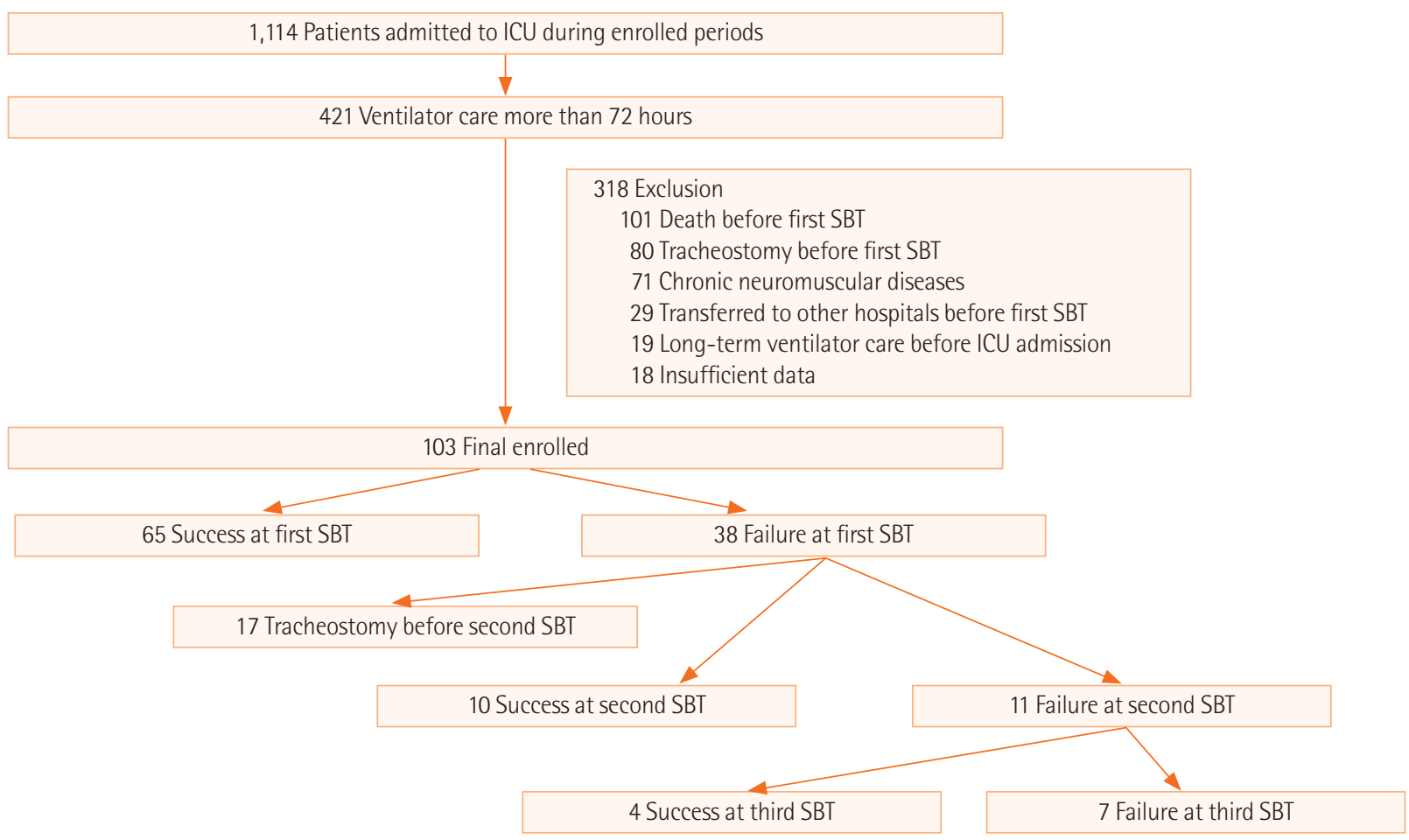

Figure 2. Flowchart of recruited and enrolled study participants and clinical outcomes of all enrolled patients. ICU: Intensive care unit; SBT: spontaneous breathing trial. 
Table 1. Comparisons of clinical characteristics between patients with final successful and unsuccessful liberation from MV

\begin{tabular}{|c|c|c|c|c|}
\hline \multirow{2}{*}{ Variable } & \multirow{2}{*}{ Total $(n=103)$} & \multicolumn{2}{|c|}{ Final liberation of MV } & \multirow{2}{*}{ P-value } \\
\hline & & Successful $(n=87)$ & Unsuccessful $(n=16)$ & \\
\hline Age (yr) & $69(22-87)$ & $69(22-86)$ & $69(45-87)$ & 0.912 \\
\hline Male sex & $72(69.9)$ & $60(69.0)$ & $12(75.0)$ & 0.771 \\
\hline $\mathrm{BMI}\left(\mathrm{kg} / \mathrm{m}^{2}\right)$ & $22.3(13.8-37.6)$ & $23.0(13.8-37.6)$ & $21.2(16.5-24.6)$ & 0.004 \\
\hline Charlson comorbidity index & $2(0-7)$ & $2(0-6)$ & $2(0-7)$ & 0.069 \\
\hline \multicolumn{5}{|l|}{ Severity of illness on day of ICU admission } \\
\hline APACHE II score & $22(10-37)$ & $22(10-37)$ & $22(11-36)$ & 0.927 \\
\hline SOFA score & $8(2-17)$ & $8(3-17)$ & $7(2-16)$ & 0.506 \\
\hline Time between ICU admission and first SBT (day) & $5(3-26)$ & $4(3-21)$ & $5(3-26)$ & 0.885 \\
\hline \multicolumn{5}{|l|}{ Underlying disease } \\
\hline Diabetes & 40 (38.8) & $37(42.5)$ & $3(18.8)$ & 0.096 \\
\hline Hemato-oncologic disease & $27(26.2)$ & $19(21.8)$ & $8(50.0)$ & 0.029 \\
\hline Chronic heart disease $^{\mathrm{a}}$ & $25(24.3)$ & $21(24.1)$ & $4(25.0)$ & $>0.999$ \\
\hline Chronic lung disease $^{b}$ & $17(16.5)$ & $16(18.4)$ & $1(6.3)$ & 0.462 \\
\hline Neurologic disease ${ }^{c}$ & $16(15.5)$ & $15(17.2)$ & $1(6.3)$ & 0.456 \\
\hline Chronic kidney disease (including hemodialysis) & $10(9.7)$ & $9(10.3)$ & $1(6.3)$ & $>0.999$ \\
\hline Alimentary disease & $9(8.7)$ & $8(9.2)$ & $1(6.3)$ & $>0.999$ \\
\hline Chronic liver disease $^{d}$ & $7(6.8)$ & $6(6.9)$ & $1(6.3)$ & $>0.999$ \\
\hline Tracheostomy during ICU stay & $30(29.1)$ & $20(23.0)$ & $10(62.5)$ & 0.003 \\
\hline ICU mortality & $19(18.4)$ & $9(10.3)$ & $10(62.5)$ & $<0.001$ \\
\hline Hospital mortality & 29 (19.5) & $17(19.5)$ & $12(75.0)$ & $<0.001$ \\
\hline
\end{tabular}

Values are presented as median (range) for continuous variables or number (\%) for categorical variables. The Mann-Whitney U-test was used for comparison of continuous variables and the chi-square or Fisher exact test (for small numbers) were used to compare categorical variables.

MV: mechanical ventilation; BMI: body mass index; ICU: intensive care unit; APACHE: Acute Physiology and Chronic Health Evaluation; SOFA: Sequential Organ Failure Assessment; SBT: spontaneous breathing trial.

${ }^{a}$ Chronic heart diseases included ischemic heart diseases, arrhythmia, and valvular heart diseases; ${ }^{b}$ Chronic lung diseases include chronic obstructive pulmonary diseases, asthma, and tuberculosis-destroyed lung; 'Neurologic diseases included stroke, neuromuscular diseases, and neurodegenerative diseases; ${ }^{\mathrm{d} C h r o n i c}$ liver diseases included hepatocellular carcinoma and liver cirrhosis.

in the final unsuccessful group had significantly lower BMIs and a higher rate of hemato-oncologic diseases as comorbidities as well as tracheostomy during their ICU stay (Table 1).

Table 2 shows the comparison between patients with successful and unsuccessful liberation of MV at the time of first SBT in all subjects. The m-BWAP score at first SBT was significantly higher in the successful group. In analyses according to all m-BWAP scoring checklist elements (Table 3) [12], the successful group had a significantly higher rate in five factors of all checklist elements when compared with the unsuccessful group (i.e., stable partial pressure of oxygen $\left[\mathrm{PaO}_{2}\right]$ and partial pressure of carbon dioxide $\left[\mathrm{PaCO}_{2}\right]$, stable pulmonary infiltration and pleural effusion, general body strength and condition, ability to clear airway, and low RSBI).

We further investigated the m-BWAP score cutoff level for predicting successful liberation from MV at first SBT, ultimate- ly identifying a value of 53, as determined by the Youden's index. Using this cutoff level, the sensitivity and specificity were determined to be $82 \%$ and $55 \%$, respectively (AUC, $0.748 ; 95 \%$ confidence interval $[\mathrm{CI}], 0.650$ to $0.847 ; \mathrm{P}<0.001)$. The corresponding positive and negative predictive values were $76 \%$ and $64 \%$, respectively. When we further compared the m-BWAP score and other traditional weaning parameters (i.e., RSBI, Pimax), the AUCs for RSBI and Pimax were 0.637 (95\% CI, 0.528 to $0.745 ; \mathrm{P}=0.014$ ) and 0.545 (95\% CI, 0.429 to $0.661 ; \mathrm{P}=0.443$ ), respectively (Figure 3 ), and we found that the AUC for m-BWAP was significantly higher than that for $\operatorname{Pimax}(\mathrm{P}=0.005)$.

\section{DISCUSSION}

To the best of our knowledge, this is the first study to investigate the clinical utility of the m-BWAP score at first SBT in Ko- 
Table 2. Comparisons of clinical characteristics between patients with successful and unsuccessful liberation of MV at first SBT

\begin{tabular}{|c|c|c|c|}
\hline Variable & Successful $(n=65)$ & Unsuccessful $(n=38)$ & P-value \\
\hline Age (yr) & $70(22-87)$ & $68(22-85)$ & 0.738 \\
\hline Male sex & 45 (69.2) & $27(71.1)$ & $>0.999$ \\
\hline BMI & $22.7(13.8-37.6)$ & $22.0(13.8-37.6)$ & 0.209 \\
\hline Charlson comorbidity index & $2(0-6)$ & $2(0-7)$ & 0.162 \\
\hline APACHE II score on day of ICU admission & $22(10-35)$ & $21(11-37)$ & 0.317 \\
\hline SOFA score on day of ICU admission & $9(3-17)$ & $8(2-16)$ & 0.421 \\
\hline Period between ICU admission to first SBT & $6(4-26)$ & $4(3-21)$ & $<0.001$ \\
\hline ICU length of stay (day) & $13(4-42)$ & $18(5-72)$ & 0.040 \\
\hline MV duration (day) & $6(4-26)$ & $10(4-71)$ & $<0.001$ \\
\hline m-BWAP score at first SBT & $60(43-80)$ & $53(33-70)$ & $<0.001$ \\
\hline $\mathrm{RSBI}$ & $42.5(6.3-93.0)$ & $50.5(22.8-150.0)$ & 0.006 \\
\hline Pimax & $16(9-32)$ & $16(10-23)$ & 0.504 \\
\hline 28-Day mortality after first SBT & $9(13.8)$ & $14(36.8)$ & 0.013 \\
\hline ICU mortality & $5(7.7)$ & 14 (36.8) & $<0.001$ \\
\hline
\end{tabular}

Values are presented as median (range) for continuous variables or number (\%) for categorical variables. The Mann-Whitney U-test was used for comparisons of the continuous variables. The chi-square or Fisher exact test (for small numbers) were used to compare categorical variables.

MV: mechanical ventilation; SBT: spontaneous breathing trial; BMI: body mass index; APACHE: Acute Physiology and Chronic Health Evaluation; ICU: Intensive care unit; SOFA: Sequential Organ Failure Assessment; m-BWAP: modified Burns Wean Assessment Program; RSBI: rapid shallow breathing index; Pimax: maximal inspiratory pressure.

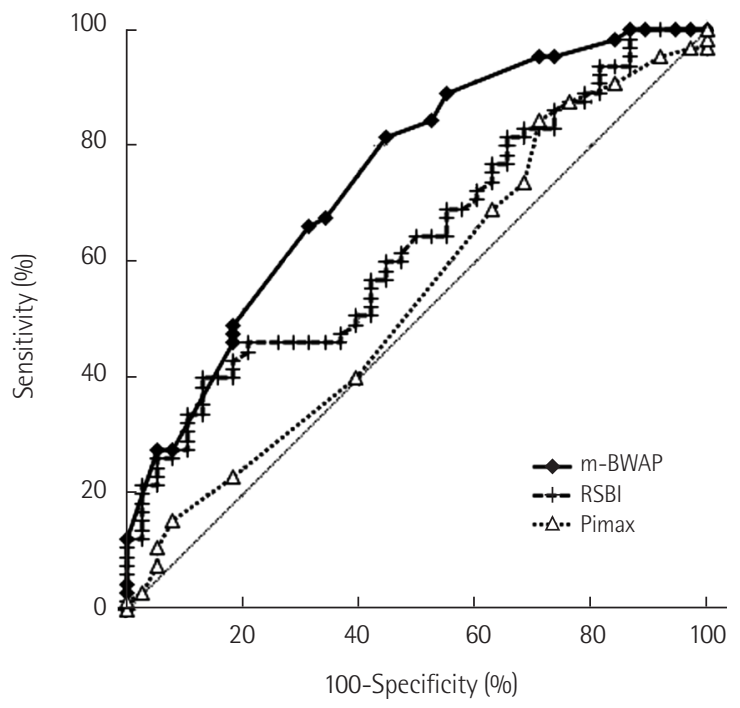

Figure 3. Receiver operating characteristic curves for modified Burns Wean Assessment Program (m-BWAP) score, rapid shallow breathing index (RSBI), and maximal inspiratory pressure (Pimax) at first spontaneous breathing trial for predicting successful liberation from mechanical ventilation were drawn. The areas under the curve (AUCs) for m-BWAP, RSBI, and Pimax were 0.748 (95\% confidence interval [Cl], 0.650 to $0.847 ; \mathrm{P}<0.001), 0.637(95 \% \mathrm{Cl}$, 0.528 to $0.745 ; \mathrm{P}=0.014)$, and $0.545(95 \% \mathrm{Cl}, 0.429$ to $0.661 ; \mathrm{P}=$ $0.443)$, respectively. The AUC for $\mathrm{m}$-BWAP was significantly higher than that for Pimax $(\mathrm{P}=0.005)$. rea. In our investigation, we found that the m-BWAP score could be successfully applied to Korean patients and determined that it is most useful in patients with an endotracheal tube at the time of first SBT. Also, the present study showed good clinical utility of the m-BWAP score at the time of first SBT to predict the likelihood of liberation from MV, regardless of the duration of MV. Our study showed m-BWAP score at first SBT was significantly higher in the successful group (Table 2). In addition, when we compared m-BWAP score with other traditional weaing indices (e.g., RSBI, Pimax), this score was not inferior for predicting liberation from MV. Our findings are very similar to those of the original m-BWAP study in the literature [12]. Although critical care resources and facilities of university hospitals in Korea are typically more limited than in Western countries $[17,18]$, our study suggests the $\mathrm{m}$ BWAP score, which was obtained by advanced practice nurses (such as critical care nurse practitioners), would be useful to predict successful liberation from MV.

In our study, the successful group showed a significant difference in five factors when compared with the unsuccessful group (e.g., stable $\mathrm{PaO}_{2}$ and $\mathrm{PaCO}_{2}$, stable pulmonary infiltration and pleural effusion, general body strength and condition, ability to clear airway, and low RSBI) (Table 3). These results suggest it is likely there are more effective factors among the m-BWAP scoring checklist elements that can predict suc- 
Table 3. Comparison of m-BWAP scoring instruments between patients who were successfully and unsuccessfully liberated from MV at the first SBT

\begin{tabular}{|c|c|c|c|}
\hline Description of category & Successful $(n=65)$ & Unsuccessful $(n=38)$ & P-value \\
\hline Age $<65$ years & $26(40.0)$ & $15(39.5)$ & $>0.999$ \\
\hline Without heart failure & $63(96.9)$ & $36(94.7)$ & 0.625 \\
\hline Stable heart rhythm & $46(70.8)$ & $24(63.2)$ & 0.513 \\
\hline Stable blood pressure & $38(58.5)$ & $21(55.3)$ & 0.837 \\
\hline Free from factors that will increase or decrease metabolic rate & 0 & 0 & $>0.999$ \\
\hline Stable arterial blood gases $\mathrm{pH}$ & $15(23.1)$ & $11(28.9)$ & 0.639 \\
\hline Stable $\mathrm{PaO}_{2}$ and $\mathrm{PaCO}_{2}$ & $60(92.3)$ & $26(68.4)$ & 0.002 \\
\hline Stable pulmonary infiltration and pleural effusion & $52(80.0)$ & $19(50.0)$ & 0.002 \\
\hline Good consciousness & $56(86.2)$ & $27(71.1)$ & 0.113 \\
\hline Stable sodium, potassium, calcium, and phosphate concentration & $3(4.6)$ & $2(5.3)$ & $>0.999$ \\
\hline Stable fluid status & $57(87.7)$ & $30(78.9)$ & 0.268 \\
\hline Good nutritional status & $17(26.2)$ & $5(13.2)$ & 0.142 \\
\hline General body strength and condition & $19(29.2)$ & $1(2.6)$ & 0.001 \\
\hline Ability to clear airway & $16(24.6)$ & $1(2.6)$ & 0.005 \\
\hline Amount and consistency of secretion & $46(70.8)$ & $24(63.2)$ & 0.513 \\
\hline Good lung compliance & $64(98.5)$ & $38(100.0)$ & $>0.999$ \\
\hline Low Raw & $61(93.8)$ & $37(97.4)$ & 0.649 \\
\hline Low Pimax & 59 (90.8) & $32(84.2)$ & 0.351 \\
\hline Low RSBI & $65(100.0)$ & $33(86.8)$ & 0.006 \\
\hline Appropriate minute ventilation & $37(56.9)$ & $16(42.1)$ & 0.159 \\
\hline
\end{tabular}

Values are presented as number (\%). This table shows the comparison between patients with successful and unsuccessful liberation of ventilator care at the time of first SBT in total patients regardless of whether they had 5 points or not according to the definition of each category.

m-BWAP: modified Burns Wean Assessment Program; MV: mechanical ventilation; SBT: spontaneous breathing trial; $\mathrm{PaO}_{2}$ : partial pressure of oxygen; $\mathrm{PaCO}_{2}$ : partial pressure of carbon dioxide; Raw: airway resistance; Pimax: maximal inspiratory pressure; RSBI: rapid shallow breathing index.

cessful liberation from MV; further research is needed to identify which factors are most useful in predicting liberation from MV.

We also attempted to evaluate the clinical usefulness of $\mathrm{m}$ BWAP score in subjects with more than two SBTs. In subjects with an endotracheal tube at first SBT $(n=103)$, a second SBT was performed in 21 subjects (20.3\%) because 17 patients underwent tracheostomy (Figure 2). Further analysis with these 21 patients showed m-BWAP score was significantly higher in the successful liberation group of MV $(n=10)$ versus the unsuccessful group (median, 60; range, 53 to 80 vs. median, 55; range, 31 to $60 ; \mathrm{P}=0.027$ ). However, we could not evaluate clinical usefulness in patients with more than three SBT attempts because of the small number of subjects. Therefore, m-BWAP score would be useful for predicting successful liberation of $\mathrm{MV}$, but further evaluation is required to determine the clinical usefulness of the m-BWAP score in subjects undergoing more than two SBTs.
In addition, we also evaluated the clinical usefulness of patients with tracheostomy $(n=80)$ before first SBT. By contrast, although m-BWAP score at first SBT was higher in subjects with tracheostomy than in those with endotracheal tubes, we identified that there was no significant difference of $m$-BWAP score between successful $(n=55)$ and unsuccessful $(n=25)$ groups (median, 65; range, 48 to 85 vs. median, 63; range, 38 to $90 ; \mathrm{P}=0.312$ ). Also, there was no predictive value for successful liberation from MV at first SBT in patients with tracheostomy (total and subgroup analysis). Patients with tracheostomy had less dead space than did those with endotracheal intubation; a new m-BWAP score for tracheostomized patients is necessary for predicting liberation from MV. Because there are no national evidence-based guidelines for the use of tracheostomy in Korea, further investigations patients at a largescale multicenter study that are applicable in Korean patients are required to develop a new weaning index for tracheostomized patients. 
The present study has several limitations. First, we could not use a randomized controlled design, which may have introduced bias. Second, the study sample size was small and the study was performed at a single center, with data obtained only from the medical ICU. Our results may not, therefore, be representative of the wider population. Third, we hypothesized that the m-BWAP score would be a more useful predictor of weaning success through subgroup analysis (such as underlying diseases, main cause of respiratory failure, and main department at ICU admission), but no significant findings were seen. Fourth, the successful group had a significantly longer period between ICU admission to first SBT (Table 2); however, we could not find the exact cause.

In conclusion, this study shows that m-BWAP scores obtained by a critical care nurse practitioner can be usefully applied to patients undergoing a weaning trial and represent a good predictor of weaning success in patients with endotracheal intubation in place at first SBT. Our results suggest that large-scale, multicenter investigations are required to further evaluate the clinical utility of m-BWAP scores in patients undergoing weaning trials.

\section{CONFLICT OF INTEREST}

No potential conflict of interest relevant to this article was reported.

\section{ACKNOWLEDGMENTS}

This work was supported by clinical research grant from the Pusan National University Hospital in 2018, Busan, Korea.

\section{ORCID}

Eun SukJeong https://orcid.org/0000-0002-6724-3942

Kwangha Lee https://orcid.org/0000-0001-9878-201X

\section{REFERENCES}

1. Combes A, Costa MA, Trouillet JL, Baudot J, Mokhtari M, Gibert C, et al. Morbidity, mortality, and quality-of-life outcomes of patients requiring $>$ or $=14$ days of mechanical ventilation. Crit Care Med 2003;31:1373-81.

2. Douglas SL, Daly BJ, Gordon N, Brennan PF. Survival and quality of life: short-term versus long-term ventilator patients. Crit Care Med 2002;30:2655-62.

3. Epstein SK. Extubation. Respir Care 2002;47:483-92.
4. Macintyre NR. Evidence-based assessments in the ventilator discontinuation process. Respir Care 2012;57:1611-8.

5. Vassilakopoulos T, Zakynthinos S, Roussos Ch. Respiratory muscles and weaning failure. Eur Respir J 1996;9:2383-400.

6. McConville JF, Kress JP. Weaning patients from the ventilator. N Engl J Med 2012;367:2233-9.

7. Jiang JR, Tsai TH, Jerng JS, Yu CJ, Wu HD, Yang PC. Ultrasonographic evaluation of liver/spleen movements and extubation outcome. Chest 2004;126:179-85.

8. Burns SM, Marshall M, Burns JE, Ryan B, Wilmoth D, Carpenter R, et al. Design, testing, and results of an outcomes-managed approach to patients requiring prolonged mechanical ventilation. Am J Crit Care 1998;7:45-57.

9. Burns SM, Earven S, Fisher C, Lewis R, Merrell P, Schubart JR, et al. Implementation of an institutional program to improve clinical and financial outcomes of mechanically ventilated patients: one-year outcomes and lessons learned. Crit Care Med 2003;31:2752-63.

10. Burns SM, Fisher C, Earven Tribble SS, Lewis R, Merrel P, Conaway MR, et al. Multifactor clinical score and outcome of mechanical ventilation weaning trials: Burns Wean Assessment Program. Am J Crit Care 2010;19:431-9.

11. Burns SM, Fisher C, Tribble SE, Lewis R, Merrel P, Conaway $\mathrm{MR}$, et al. The relationship of 26 clinical factors to weaning outcome. Am J Crit Care 2012;21:52-8.

12. Jiang JR, Yen SY, Chien JY, Liu HC, Wu YL, Chen CH. Predicting weaning and extubation outcomes in long-term mechanically ventilated patients using the modified Burns Wean Assessment Program scores. Respirology 2014;19:576-82.

13. Roh JH, Synn A, Lim CM, Suh HJ, Hong SB, Huh JW, et al. A weaning protocol administered by critical care nurses for the weaning of patients from mechanical ventilation. J Crit Care 2012;27:549-55

14. Tonnelier JM, Prat G, Le Gal G, Gut-Gobert C, Renault A, Boles JM, et al. Impact of a nurses' protocol-directed weaning procedure on outcomes in patients undergoing mechanical ventilation for longer than 48 hours: a prospective cohort study with a matched historical control group. Crit Care 2005;9:R83-9.

15. Danckers M, Grosu H, Jean R, Cruz RB, Fidellaga A, Han Q, et al. Nurse-driven, protocol-directed weaning from mechanical ventilation improves clinical outcomes and is well accepted by intensive care unit physicians. J Crit Care 2013;28:43341.

16. Fagoni N, Piva S, Peli E, Turla F, Pecci E, Gualdoni L, et al. Comparison between a nurse-led weaning protocol and weaning based on physician's clinical judgment in tracheostomized critically ill patients: a pilot randomized controlled clinical 
trial. Ann Intensive Care 2018;8:11.

17. Kim JH, Hong SK, Kim KC, Lee MG, Lee KM, Jung SS, et al. Influence of full-time intensivist and the nurse-to-patient ratio on the implementation of severe sepsis bundles in Korean intensive care units. J Crit Care 2012;27:414.e11-21.

18. Kwak SH, Jeong CW, Lee SH, Lee HJ, Koh Y. Current status of intensive care units registered as critical care subspecialty training hospitals in Korea. J Korean Med Sci 2014;29:431-7.

19. Acute Respiratory Distress Syndrome Network, Brower RG, Matthay MA, Morris A, Schoenfeld D, Thompson BT, et al. Ventilation with lower tidal volumes as compared with traditional tidal volumes for acute lung injury and the acute respiratory distress syndrome. N Engl J Med 2000;342:1301-8.

20. MacIntyre NR. The ventilator discontinuation process: an expanding evidence base. Respir Care 2013;58:1074-86.

21. Haas CF, Loik PS. Ventilator discontinuation protocols. Respir Care 2012;57:1649-62.

22. Boles JM, Bion J, Connors A, Herridge M, Marsh B, Melot C, et al. Weaning from mechanical ventilation. Eur Respir J 2007; 29:1033-56

23. Charlson ME, Pompei P, Ales KL, MacKenzie CR. A new method of classifying prognostic comorbidity in longitudinal studies: development and validation. J Chronic Dis 1987;40:37383.

24. Knaus WA, Draper EA, Wagner DP, Zimmerman JE. APACHE II: a severity of disease classification system. Crit Care Med $1985 ; 13: 818-29$.

25. Vincent JL, Moreno R, Takala J, Willatts S, De Mendonça A, Bruining $\mathrm{H}$, et al. The SOFA (Sepsis-related Organ Failure Assessment) score to describe organ dysfunction/failure. On behalf of the Working Group on Sepsis-Related Problems of the European Society of Intensive Care Medicine. Intensive Care Med 1996;22:707-10.

26. Perkins NJ, Schisterman EF. The inconsistency of "optimal" cutpoints obtained using two criteria based on the receiver operating characteristic curve. Am J Epidemiol 2006;163:670-5. 